Acta Cryst. (2002). A58 (Supplement), C75

SINGLE CRYSTAL DIFFRACTOMETER FOR CONTROL OF WAFER SHEAR PLANES AGAINST SPECIFIED CRYSTAL PLANE A.D. Zvonkov N.S. Ibraimov S.V. Nikitina C. Likhoushina D. Malagodi S Srinivasan

Institute For Roentgen Optics 1st Volokolamsky Pr. 10, PMZR MOSCOW 123060 RUSSIA

The main element of the X-ray optic layout in the portable diffractometer is Kumakhov's focusing capillary lens with a specific X-ray convergence angle of $1 \sim 3^{\circ}$ enabling, with the help of a linear-coordinate detector to register diffracted reflection at an angle of $\sim 5^{\circ}$, to determine wafer crystallographic orientation compared to a standard sample.

Requirements of the X-ray angle of convergence $\alpha$ from Kumakhov's full lens are determined by the maximum permissible deviation of the crystal plane angle $\Delta \varphi_{\max }$ from the specified angle $\varphi\left(\alpha>2 \Delta \varphi_{\max }\right)$. X-ray beam converging on the sample makes it possible to eliminate the need for sample holder rotation in search for diffraction reflection maximum.

A special sample holder has been developed to achieve precise determination of the deviation angle of wafer shear planes from the standard sample. The accuracy of the developed comparator diffractometer in regards to the above mentioned measurement depends on wafer flatness and equals to \pm 5 Åsec. Dependence of the reflected diffraction shape and width on the quality of wafer treatment during various technological processes, such as cutting, grinding, polishing, was established. The device is equipped with software for automatic determination of the angle of deviation for the wafers under analysis.

Keywords: CAPILLARY OPTICS, SINGLE CRYSTAL, WAFERS

Acta Cryst. (2002). A58 (Supplement), C75

\section{A SCATTERING USERS' BILL OF RIGHTS}

$\underline{\text { J. Barnes }}$

JDB Science LLC 7710 Chatham Rd CHEVY CHASE MARYLAND 20815-5057 USA

Many designers of scattering facilities fail to take proper account of the needs of end users when constructing their beam lines. The user has certain inalienable rights, these include: (1) The right to understand how the experiment works when it is launched. (2) Relief from the drudgery of hand recording and transcription of metadata (3) The right to data sets that are well documented, self-describing, and self-contained (4) Freedom to inspect the data in a simple and straightforward manner (5) The right to assistance in the form of tools that permit reduction and visualization of the data with minimal outside assistance. Examples of data sets that illustrate both the good and the bad implementations of these principles will be displayed.

\section{Keywords: DATA FORMAT DATA ACQUISITION DATA} REDUCTION
Acta Cryst. (2002). A58 (Supplement), C75

\section{A SCANNING MICROSCOPE FOR HARD SYNCHROTRON X-} RADIATION

M. Moore $^{1}$ V T Petrashov ${ }^{1}$ D Laundy $^{2}$ R Shaikhaidarov ${ }^{1}$ J J Allerton ${ }^{1}$ ${ }^{1}$ Dept of Physics, Royal Holloway University of London, Egham, Surrey, TW20 0EX, UK ${ }^{2}$ CLRC Daresbury Laboratory, Warrington, WA4 4AD, UK

A high-resolution scanning X-ray microscope has been made for use with hard synchrotron radiation: (energy range 6 to $60 \mathrm{keV}$, wavelength range 0.2 to 0.02 $\mathrm{nm}$ ). The collimator assembly consists of a series of parallel channels in gold of various widths, one of which being chosen for use. They have length typically $5 \mathrm{~mm}$. The dimensions of the smallest successful collimator so far made are $500 \mathrm{~nm}$ high by $300 \mathrm{~nm}$ wide: (a world record). Having a width-tolength ratio of about $1: 10000$, they have to be accurately oriented to the incident synchrotron beam. This is achieved by starting with the widest collimator $(10 \mu \mathrm{m})$ and making small angular adjustments with 'picomotors'. Then the assembly is translated to bring one of the narrower collimators into the beam. The resolution is given by the size of the collimator and the working distance is many centimetres (before diffraction effects become noticeable). The specimen is mounted on a piezo-electric X-Y scanning stage, which has a range of $0.2 \mathrm{~mm} \times 0.2 \mathrm{~mm}$ and a minimum step size of $40 \mathrm{~nm}$. An image is acquired line by line. This can be in absorption (with the possibility of extended X-ray absorption fine-structure EXAFS), in photoluminescence or in diffraction. Applications include the examination of nanostructures, the determination of structures of small crystals and studies of biological and mineralogical specimens.

\section{Keywords: MICROSCOPE, SYNCHROTRON RADIATION, X-RAY IMAGING}

\author{
Acta Cryst. (2002). A58 (Supplement), C75 \\ X-RAY DIFFRACTION SCREENING TECHNIQUES FOR \\ BIOLOGICAL SINGLE CRYSTALS

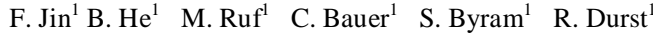 \\ Bruker-AXS
}

It is a common practice that protein crystals are first screened by a laboratory $\mathrm{X}$-ray diffraction system, and then selected crystals retrieved and transferred to synchrotron facilities for further study. There has been a great interest in the development of techniques to automatically screen large numbers of samples without human operator intervention. We describe new hardware and software developments, which facilitate this process. Hardware developments include a new robotic system with improved cryogenic sample handing, which suppresses problems with icing seen in earlier robot designs. Innovations in high-brilliancy lab x-ray source design and high sensitivity two-dimensional detectors greatly decrease the time required to screen each sample. New software algorithms for automatic sample alignment, evaluation and tracking are also described.

\section{Keywords: X-RAY BIO SCREENING SINGLE CRYSTAL}

